\title{
Efeito do extrato da Passiflora edulis na cicatrização de gastrorrafias em ratos: estudo morfológico e tensiométrico ${ }^{1}$
}

\author{
Extract of Passiflora edulis in the healing process of gastric sutures en rats: a morphological \\ and tensiometric study
}

José Ribamar Sousa da Silva ${ }^{2}$, Antonio Carlos Ligocki Campos ${ }^{3}$, Lydia Masako Ferreira ${ }^{3}$, Ayrton Alves Aranha Júnior ${ }^{2}$, Arnulf Thiede ${ }^{3}$, Luiz Alberto Zago Filho ${ }^{4}$, Lyrio César Bertoli ${ }^{5}$, Marcelo Ferreira ${ }^{5}$, Paula Suzin Trubian ${ }^{5}$, Alexandre Coutinho Teixeira de Freitas ${ }^{3}$

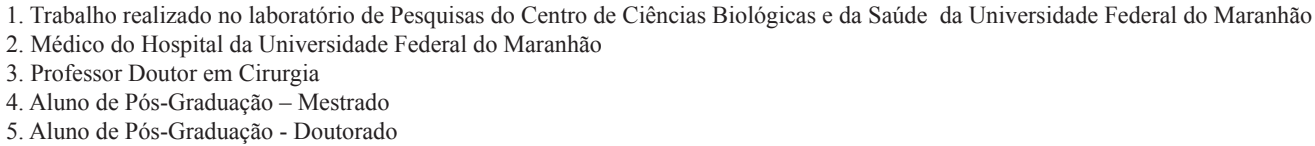

\section{RESUMO}

Introdução: Muitas substâncias de origem vegetal são utilizadas desde os primórdios da civilização com finalidade de melhorar a cicatrização. Dentre elas, foi demonstrado em ratos que o extrato de folhas secas de Passiflora edulis tem efeito antiinflamatório. Objetivo: Investigar o efeito do extrato de folhas secas de Passiflora edulis na cicatrização das gastrorrafias em ratos. Métodos: Foram utilizados 40 ratos Wistar, machos, adultos, divididos em dois grupos de 20 denominados grupo Controle e grupo Passiflora (GP e GC) subdivididos em grupos de 10 de acordo com o momento do óbito, no $3^{\circ}$ e $7^{\circ}$ dias de pós-operatório. Todos os animais foram submetidos a laparotomia mediana, na qual foi realizada gastrotomia na parede anterior do corpo seguida da gastrorrafia com fio de polipropileno 6.0, utilizando-se quatro pontos separados, em plano único total. Os ratos do GP receberam na cavidade abdominal, antes do fechamento desta, solução de extrato de Passiflora edulis, na concentração de $250 \mathrm{mg} / \mathrm{Kg} /$ peso, e os animais do GC igual volume de solução salina isotônica. Foram avaliados aspectos macroscópicos (grau de aderência de Knightly), pressão de ruptura por manômetro eletrônico e parâmetros inflamatórios microscópicos. Resultados: Todos os animais demonstraram boa cicatrização da parede abdominal, sem sinais clínicos de infecção ou deiscência. Não houve diferença estatisticamente significante em relação ao grau de aderências das gastrorrafias nos dois grupos, no $3^{\circ}$ dia $(p=0,734)$ e no $7^{\circ}$ dia $(p=1.000)$ e nem na comparação do aspecto da mucosa gástrica, no $3^{\circ}$ dia e $7^{\circ}$ dias (NS). As gastrorrafias apresentaram vazamentos com menor pressão de insuflação nos grupos de animais do $3^{\circ}$ dia em ambos os grupos. Não houve diferença significante de pressão de ruptura entre os subgrupos do $3^{\circ}$ dia $(\mathrm{GC} 3=41,1 \pm 22,1 \mathrm{mmHg}$ vs GP3 $=59,2 \pm 20,4 \mathrm{mmHg} ; \mathrm{p}=0,074)$. No $7^{\circ}$ dia houve maior média de pressão de ruptura, porém sem diferença estatisticamente significante entre os dois grupos $(\mathrm{p}=0,850)$. Eles, no $3^{\circ}$ e $7^{\circ}$ dias, não demonstraram diferenças estatisticamente significantes quanto à análise histológica, exceto em relação a proliferação fibroblástica no $7^{\circ}$ dia, do grupo GP, que apresentou maior densidade de fibroblastos nesse período $(\mathrm{p}=0,002)$. Conclusão: O uso intraperitoneal do extrato de Passiflora edulis influencia favoravelmente a cicatrização das gastrorrafias em ratos pelo aumento da proliferação fibroblástica no $7^{\circ}$ dia de pós-operatório.

Descritores: Passiflora edulis. Cicatrização de Feridas. Ruptura. Ratos.

\begin{abstract}
Introduction: Many substances of vegetable origin have been used since the beginning of civilization with the purpose of improving the healing process. Among them, dry leaves extract from Passiflora edulis have been shown to have an anti-inflammatory effect in rats. Purpose: To analyze the effect of dry leaves extract from Passiflora edulis in the healing of gastric sutures in rats. Methods: Forty male adult Wistar rats were divided into two groups of 20 rats, called Passiflora group (GP) and Control group (GC) which were divided into two groups of 10 according to moment of death, on day 3 or day 7 after the operation. All animals were submitted to a midline incision and a gastrotomy was performed on the anterior wall of the stomach followed by gastric suture with polypropylene 6.0 using four stitches on a single layer. Rats from GP were given, before closure of the abdominal wall, a solution of Passiflora edulis extract, $250 \mathrm{mg} / \mathrm{kg} / \mathrm{weight}$, while rats from the GC were given an isovolumetric isotonic saline solution. Macroscopic evaluation included the adhesion index proposed by Knightly. Bursting pressure was measure by an electronic device. Microscopic analysis was performed including inflammation parameters. Results: All animals presented adequate healing of the abdominal wall with no clinical signs of infections or dehiscence. The adherence index was similar in both groups both on day $3(p=0.734)$ and on day $7(p=1.000)$. The gastric sutures presented leak with smaller insufflation pressure on the $3^{\text {rd }}$ P.O. day in both groups as compared to the $3^{\text {th }}$ P.O. day. There was no significant difference of bursting pressure among the subgroups on the $3^{\text {rd }}$ P.O. day $(\mathrm{GC} 3=41.1 \pm$
\end{abstract}


$22.1 \mathrm{mmHg}$ versus GP3 $=59.2 \pm 20.4 \mathrm{mmHg} ; \mathrm{p}=0.074)$. On the $7^{\text {th }}$ P.O. day, there was an increased mean bursting pressure in both groups, but there was no statistically significant difference between the two groups $(\mathrm{p}=0.850)$. Histologic parameters were similar in both groups, on P.O. days 3 and day 7, except for the fibroblastic proliferation, which was greater on the 7th day in GP ( $p=0.002)$. Conclusion: The intraperitoneal use of Passiflora edulis extract influences favorably the healing of gastric sutures in rats because of the increase in the fibroblastic proliferation on the $7^{\text {th }}$ P.O. day.

Key words: Passiflora edulis. Wound Healing. Rupture. Rats.

\section{Introdução}

A história da cicatrização das feridas e o emprego da fitoterapia na sua reparação são tão antigos quanto a história da humanidade. Na verdade, do ponto de vista biológico, a lesão tecidual e suas seqüelas participam na maioria dos problemas médicos ${ }^{1}$. A resposta inflamatória é estreitamente entrelaçada com o processo de reparação ${ }^{2}$. É um componente importante e necessário para o processo de cicatrização de órgãos e tecidos.

O conhecimento das anastomoses viscerais, em particular no estômago, desenvolveu-se gradualmente de um nível empírico para um nível científico, ao longo dos últimos dois séculos. Durante essa evolução, foi dada muita ênfase aos materiais e métodos de sutura, sem muita compreensão do processo de cicatrização propriamente dito.

Durante as últimas duas décadas houve grande modificação nas indicações de procedimentos cirúrgicos sobre o estômago. Com o advento dos bloqueadores farmacológicos de acidez gástrica e o emprego de antiinfecciosos para a erradicação do Helicobacter pylori, a operação para tratamento de úlcera cloridro-péptica, que era a alternativa preferencial, ficou reservada para as emergências. Para o tratamento do câncer gástrico, que é a segunda causa de morte por neoplasia maligna do mundo, sendo superado apenas pelo câncer de pulmão, as ressecções gástricas permanecem sendo a base do tratamento.

Atualmente, com o desenvolvimento da cirurgia bariátrica, as anastomoses gástricas estão entre os procedimentos cirúrgicos mais freqüentes, inclusive realizados por videolaparoscopia. As anastomoses gástricas têm grande importância clínica pelo fato de que extravasamento do conteúdo gástrico e deiscência anastomótica continuam sendo problemas freqüentes e graves associados à alta mortalidade pós-operatória, maior permanência nos hospitais e maior custo de tratamento.

A utilização de plantas medicinais é prática que data de mais de 2.500 anos, muitas destas mencionadas em textos chineses ou ensinamentos budistas. Na natureza existem milhares de plantas com ação farmacológica. O Brasil, nesse aspecto, caracteriza-se por possuir grande riqueza de plantas, principalmente na Amazônia, que "é um viveiro admirável e inesgotável de plantas medicinais", apesar de a maioria delas ser utilizada sem embasamento científico.

Dentre os vários fitoterápicos utilizados pela população, é a espécie Passiflora edulis uma das mais conhecidas, que pertence à família Passifloraceas, originária das regiões tropicais e subtropicais do continente americano e abundante no norte do Brasil. Entre as substâncias químicas descritas na Passiflora edulis encontram-se os alcalóides (harmana, harmina, harmalina e harmol), flavanóides e carotenóides.
Vários autores constataram, na espécie Passiflora edulis (conhecida popularmente como maracujá), diversos efeitos farmacológicos. Tem sido utilizada para tratamento de afecções do sistema nervoso com ações hipnóticas, sedativas, anti - helmíntica, parassimpaticolítica, antipirética, além de propriedades analgésicas e antiinflamatórias. Em particular, tem sido demonstrado que a Passiflora edulis tem efeito analgésico e antiinflamatório, com características dos antiinflamatórios não esteróides (AINES), que diminuem a dor por inibição da biossíntese de eicosanóides, mediadores de processos inflamatórios, em geral na forma de extrato de Passiflora edulis, como chás ou topicamente.

Sabendo-se que os antiinflamatórios exercem efeito favorável sobre a cicatrização anastomótica, por reduzirem a colagenólise nos três primeiros dias, e por aumentarem a produção de colágeno ${ }^{3}$ e considerando-se que a Passiflora edulis possui efeito antiinflamatório, o objetivo deste trabalho é avaliar os efeitos do extrato da Passiflora edulis na cicatrização de gastrorrafias, em ratos.

\section{Métodos}

Este trabalho foi realizado no Laboratório de Pesquisa do Departamento de Fisiologia e Farmacologia da Universidade Federal do Maranhão, respeitando-se a Legislação Brasileira de Animais de Experimentação regulamentada pela Lei Federal 6.638 (1979). Todo o experimento obedeceu aos princípios éticos em experimentação animal, preconizados pelo Colégio Brasileiro de Experimentação Animal (COBEA). Este trabalho foi aprovado pelo Comitê de Ética do Departamento de Cirurgia do Hospital de Clínicas da Universidade Federal do Maranhão.

\section{Material}

Foram utilizados 40 ratos (Rattus norvergicus albinus, Rodentia mammalia), da linhagem Wistar, machos, com peso corporal médio de $138 \mathrm{~g}( \pm 10 \mathrm{~g})$, de 50 a 60 dias de vida adquiridos no Centro Multidisciplinar para Investigação Biológica (CEMIB) da Universidade Estadual de Campinas - São Paulo (UNICAMP) e transferidos por via aérea para o laboratório de pesquisa do Departamento de Fisiologia e Farmacologia do Centro de Ciências Biológicas e da Saúde da Universidade Federal do Maranhão (UFMA). Os animais ficaram aclimatizados por sete dias antes de serem incluídos no estudo, e receberam água e ração própria para a espécie (Purina $^{\circledR}$, Labina, São Paulo, SP ) ad libitum.

\section{Preparo do extrato da Passiflora edulis}

Foram coletados cerca de $8 \mathrm{~kg}$ de folhas íntegras de Passiflora edulis. O material foi identificado e encontra- 
se catalogado no Herbário Ático Seabra, da Universidade Federal do Maranhão, sob o número 1155. Da parte aérea da planta Passiflora edulis retiraram-se as folhas, que foram colocadas à temperatura ambiente para secar durante duas semanas. Em seguida, esse material foi colocado em estufa de secagem para a retirada da umidade em temperatura que variou de $45^{\circ}$ a $50^{\circ} \mathrm{C}$ por $24 \mathrm{~h}$ e, posteriormente, foi submetido ao processo de moagem em moinho elétrico (Tecnal TE - $650^{\circledR}$, São Paulo, SP). Foram obtidos 2000 gramas de um pó de coloração esverdeada. Foram adicionados seis litros de solução hidroalcoólica a $70 \%$, na proporção de $1: 3$. A mistura permaneceu por $12 \mathrm{~h}$ sendo que a cada $2 \mathrm{~h}$ agitava-se por cinco minutos. Depois foi filtrada em funil de vidro simples e algodão. Os procedimentos foram repetidos por mais duas vezes, obtendo-se, no final dessa filtração, quantidade de $5500 \mathrm{ml}$ de extrato bruto de coloração verdeescuro. O extrato bruto foi concentrado em evaporador rotativo (Fisaton $802^{\circledR}$, São Paulo, SP), a temperatura de $50-55^{\circ} \mathrm{C}$, para a eliminação total do solvente. $\mathrm{O}$ material obtido, depois da concentração, apresentou-se em forma de pasta. Deste foram retiradas as amostras para os testes farmacológicos no Laboratório de Bioquímica do Centro de Ciências Biológicas e da Saúde da UFMA.

Para a determinação da concentração $(\mathrm{g} / \mathrm{ml})$ do extrato, foi calculado o seu peso seco. Em três beckeres de peso determinado, adicionou-se $1 \mathrm{ml}$ do extrato em cada um, em seguida evaporou-se o solvente em corrente de ar quente. Os pesos das amostras foram posteriormente determinados em balança analítica digital (modelo AL-200 ${ }^{\circledR}$ ) no Laboratório de Produtos Naturais da UFMA e repetidos por três vezes até estabilização dos valores. A média aritmética dos pesos dos beckeres correspondeu ao peso seco do extrato. Para os testes farmacológicos intraperitoneais, foram retirados $25 \mathrm{~g}$ do extrato bruto hidroalcoólico da Passiflora e diluiu-se em $100 \mathrm{ml}$ da solução salina, o que resultou em concentração de $250 \mathrm{mg} / \mathrm{ml}$.

A análise da composição físico-química do extrato de Passiflora edulis foi realizada no Laboratório de Controle de Qualidade de Alimentos e Águas do Departamento de Tecnologia Química da UFMA. Na análise de $50 \mathrm{ml} \mathrm{da}$ solução do extrato de Passiflora edulis foram encontradas as seguintes substâncias: proteínas: 9,98\%; carbohidratos: 19,2\%; lipídeos: 6,54\%; cinzas: 5,65\%; umidade: 58,63\%; $\mathrm{pH}: 5,1$.

\section{Métodos}

Os ratos foram distribuídos aleatoriamente em dois grupos de 20, conforme o uso ou não de extrato. O grupo Passiflora (GP) recebeu o extrato hidroalcoólico de Passiflora edulis, e o grupo Controle (GC) recebeu quantidade isovolumétrica de solução salina isotônica (Figura 1). Cada grupo foi dividido em dois subgrupos com 10 ratos cada, conforme especificado a seguir o dia do óbito: $3^{\circ}$ ou $7^{\circ}$ dia pós-operatório.

\section{Procedimento cirúrgico}

Após o jejum de 12 horas, com acesso livre à água, os ratos foram submetidos à anestesia geral inalatória com éter sulfúrico comercial a 50\%, em campânula de plástico. Durante o procedimento cirúrgico foi mantida anestesia inalatória em sistema semi - aberto. A dose anestésica durante a operação foi baseada na observação da amplitude e freqüência dos movimentos respiratórios.

Após a anestesia cada animal foi pesado em balança eletrônica, modelo A 500, marca Mart (Balança e Aparelhos de Precisão Ltda., Indústria Brasileira, São Paulo, S.P). A seguir, procedeu-se à fixação do mesmo em prancha cirúrgica, posicionando-o em decúbito dorsal, com os membros em extensão e contidos por tensores elásticos. Foi realizada a tricotomia do terço superior do abdômen $\left(4,0 \mathrm{~cm}^{2}\right)$, rente à pele, com lâmina de barbear. A degermação da pele foi feita com solução degermante de polivinilpirrolidona-Iodo (Riodeine Degermante ${ }^{\circledR}$, Rioquímica, São José do Rio Preto, SP) e posterior anti-sepsia abdominal com polivinilpirrolidona - Iodo a 10\% (Riodeine Tópico ${ }^{\circledR}$, Rioquímica, São José do Rio Preto, SP) e a colocação de campo fenestrado esterilizado sobre o animal, expondo a região abdominal. Obteve-se acesso à cavidade abdominal, mediante incisão mediana de $3 \mathrm{~cm}$ de extensão. Após a exposição do estômago, efetuou-se incisão longitudinal de $1,0 \mathrm{~cm}$ de comprimento no maior eixo da parede anterior do corpo gástrico, próximo à grande curvatura, envolvendo todos os planos. A gastrorrafia foi realizada com fio de polipropileno (Prolene ${ }^{\circledR}$, Ethicon, São José dos Campos, São Paulo), 6-0, utilizando-se quatro pontos separados, interessando plano único total (Figura 1).

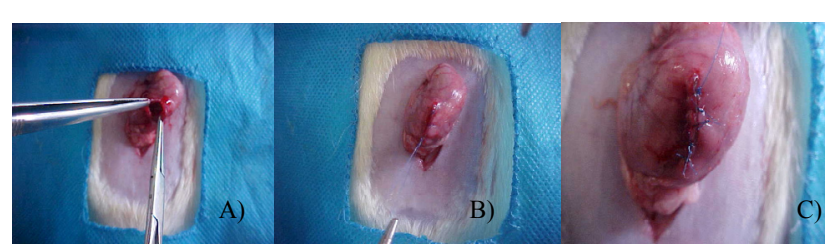

FIGURA 1 - Exposição e abertura do estômago com posterior gastrorrafia. Nota: A) Exposição e abertura do estômago; B) e C) Sutura do estômago.

A seguir, aplicou-se, na cavidade abdominal, solução de extrato de Passiflora edulis, na concentração de $250 \mathrm{mg} / \mathrm{kg} /$ peso, ou igual volume de solução salina isotônica, conforme o grupo em estudo, utilizando seringa de $1 \mathrm{ml}$. O fechamento da parede foi realizado com fio monofilamentar, inabsorvível (Mononylon $^{\circledR}$, Ethicon, São José dos Campos, São Paulo, 5.0) (Figura 2).

Após a recuperação anestésica cada rato foi recolocado em sua gaiola de origem, onde voltou a receber, após uma hora, água e ração balanceada para a espécie, e permaneceu sob condições já citadas de temperatura e iluminação, anotando-se condições gerais das feridas e possíveis intercorrências em fichas de protocolo individual. 

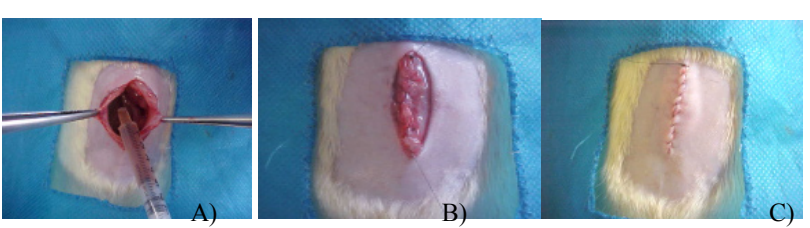

FIGURA 2 - Processo de colocação do extrato de Passiflora edulis na cavidade operatória, com posterior fechamento da parede abdominal. A) Colocação do extrato na cavidade abdominal; B) e C) Fechamento da parede abdominal

\section{Sacrificio dos animais}

Os ratos foram mortos por dose letal inalatória de éter sulfúrico. A abertura da cavidade abdominal foi feita mediante duas incisões transversais (uma abaixo do processo xifóide e outra acima do púbis) e outra longitudinal, paralela à cicatriz mediana decorrente do ato operatório prévio. $\mathrm{Na}$ inspeção da cavidade abdominal, verificou-se a presença de aderências, sinais de infecção, a integridade da gastrorrafia. Após, retirou-se todo o estômago do animal, seccionandose o duodeno a $0,6 \mathrm{~cm}$ do piloro, o esôfago a $0,6 \mathrm{~cm}$ da junção esofagogástrica, além de seccionarem-se ligamentos do estômago com o baço e fígado. As estruturas ou órgãos aderidos à gastrorrafia eram retirados cuidadosamente, em monobloco (Figura 3).

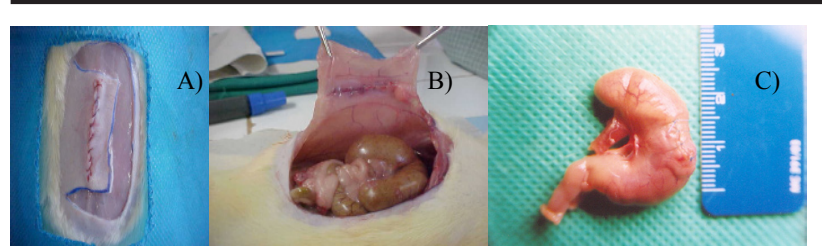

FIGURA 3 - Sítio cirúrgico. A) e B) Abertura da cavidade abdominal para retirada do estômago; C) Exposição do estômago após retirada da cavidade abdominal

\section{Avaliação macroscópica}

Inspecionou-se a cavidade abdominal para a ocorrência de aderências, complicações da gastrorrafia e infecção intra-abdominal. A intensidade de aderências ao redor da gastrorrafia foi analisada segundo os critérios quantificados pelo índice de Knigthly ${ }^{4}$ (Figura 4).

\section{Teste de resistência à insuflação de ar atmosférico}

A avaliação do teste de resistência à insuflação do ar atmosférico foi realizada segundo a metodologia proposta por Warde $^{5}$ e Czeczko ${ }^{6}$, obedecendo-se as seguintes etapas:

a) preparo da peça cirúrgica, mantendo-se as estruturas aderidas a ela;

b) apreensão do segmento distal com pinça tipo Kelly; c) introdução de uma sonda de Nelaton ${ }^{\circledR}$, de calibre $n^{\circ}$ 06 na porção distal do esôfago por $0,5 \mathrm{~cm}$ e ligadura deste sobre a sonda com fio de algodão $2-0$ (Polycot ${ }^{\mathbb{R}}$, Ethicon, New Jersey, EUA);

d) conexão da sonda a uma bomba de infusão (Polzin ${ }^{\circledR}$, Watson Marlow, Alemanha), que foi regulada para insuflação gradativa de ar ambiente à velocidade de $0,1 \mathrm{ml} / \mathrm{s}$. A pressão foi registrada pelo manômetro em mmHg (Figura 5);

e) submersão da peça em recipiente de vidro contendo água;

f) insuflação gradativa de ar ambiente até a ocorrência de liberação de bolhas de ar, caracterizando o momento de ruptura da peça, sendo registrado o local da ruptura e a pressão aferida pelo manômetro.

\begin{tabular}{|c|c|}
\hline Grau & Característica Ausência de aderências \\
\hline 1 & Aderência única, fina e facilmente separável. \\
\hline 2 & $\begin{array}{l}\text { Aderências em pequena extensão, fracas e que se } \\
\text { rompem com pequena tração. }\end{array}$ \\
\hline 3 & $\begin{array}{l}\text { Aderências viscerais extensas que se estendem } \\
\text { até a parede }\end{array}$ \\
\hline 4 & $\begin{array}{l}\text { Aderências numerosas, extensas e densas que en- } \\
\text { volvem o mesentério, intestino, omento e parede } \\
\text { abdominal }\end{array}$ \\
\hline
\end{tabular}

FIGURA 4 - Índice de Knigthly para mensuração da intensidade das aderências

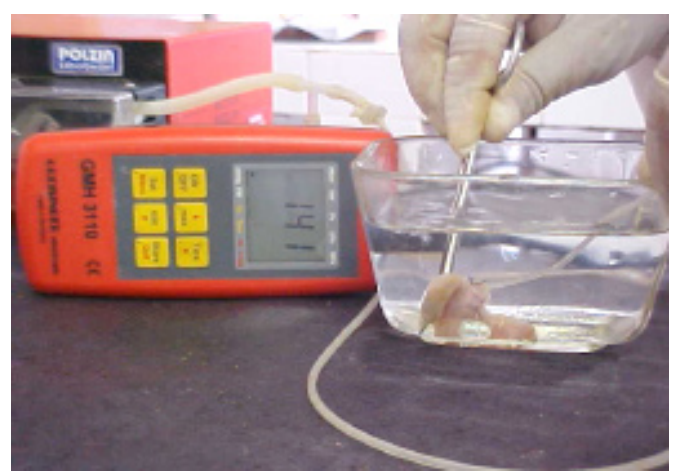

FIGURA 5 - Teste de resistência da gastrorrafia à insuflação do ar

\section{Avaliação microscópica}

As peças foram fixadas em placas de isopor, identificadas e acondicionadas em recipientes contendo formol a $10 \%$. O processamento histológico e a análise das lâminas seguiram a rotina do Serviço de Anatomia Patológica do Hospital da Universidade Federal do Maranhão. Procedeuse a técnica histológica de rotina, corados pela técnica de hematoxilina-eosina.

A análise dos cortes histológicos foi realizada em microscópio tetraocular (OLYMPUS modelo CH-30, New York, EUA), sem o conhecimento prévio pelo patologista do grupo a que pertencia o animal. O campo histológico 
avaliado foi a área de sutura, examinando-se oito campos de cada lâmina, utilizando as objetivas com aumento de quatro, 10 e 40 vezes, e ocular de 10 vezes.

Os critérios histológicos incluíram: inflamação aguda, inflamação crônica inespecífica, necrose isquêmica, reação gigantocelular do tipo corpo estranho, proliferação fibroblástica, fibrose (colagenização), reepitelização, coaptação das bordas da sutura, neoformação capilar e extensão da inflamação segundo Cotran ${ }^{7}$.

\section{Análise estatística}

Os dados foram analisados utilizando-se o programa Statistica for Windows 5.1. A pressão de insuflação de ar foi analisada pelo teste $t$ de Student. As variáveis das avaliações macroscópica e microscópica foram analisadas pelo teste não-paramétrico de Mann-Whitney. Em todos os testes foram feitas análises intergrupo (Controle e Passiflora) e intragrupo (Controle (GC3) - Controle (GC7); Passiflora (GP3) - Passiflora (GP7)). O nível de significância (a) utilizado para se rejeitar a hipótese da nulidade foi de 0,05 .

\section{Resultado}

$\mathrm{O}$ ato operatório de todos os animais transcorreu sem complicações. Não houve óbitos. Todos os animais recuperaram-se bem da anestesia; durante o período pós-operatório não houve intercorrências. As avaliações clínicas diárias mostraram recuperação satisfatória, com manutenção do estado geral, presença de atividade física e disposição para alimentar-se.

\section{Avaliação macroscópica}

A comparação da freqüência das aderências conforme os critérios de quantificação do índice de Knigthly ${ }^{4}$, estão relatados na Tabela 1 . Não foram evidenciadas diferenças entre os grupos.

TABELA 1 - Distribuição dos animais de acordo com os grupos em estudo (Controle (GC) e Passiflora edulis (GP)) quanto ao índice de Knigthly, no $3^{\circ}$ e $7^{\circ}$ dia de P.O.

\begin{tabular}{|c|c|c|c|c|c|c|}
\hline \multirow{2}{*}{$\begin{array}{l}\text { Índice de } \\
\text { Knigthly }\end{array}$} & \multicolumn{2}{|c|}{ Controle } & \multicolumn{2}{|c|}{ Passiflora } & \multicolumn{2}{|c|}{$\mathbf{p}$} \\
\hline & 3 dias & 7 dias & 3 dias & 7 dias & GC3 $\times$ GP3 & GC7 $\times$ GP7 \\
\hline $\begin{array}{l}\text { Grau de } \\
\text { Aderência }\end{array}$ & & & & & 0,733 & 1,000 \\
\hline 0 & 3 & 1 & 1 & 0 & & \\
\hline 1 & 5 & 8 & 7 & 10 & & \\
\hline 2 & 2 & 1 & 2 & 0 & & \\
\hline 3 & 0 & 0 & 0 & 0 & & \\
\hline 4 & 0 & 0 & 0 & 0 & & \\
\hline Total & 10 & 10 & 10 & 10 & & \\
\hline
\end{tabular}

p - Nível de significância estatística

\section{Teste de resistência à insuflação de ar atmosférico}

No teste de resistência à insuflação de ar, no $3^{\circ}$ e $7^{\circ}$ dias de pós-operatórios, o vazamento de ar ocorreu na linha de sutura em todos os animais. Comparando-se os tempos de avaliação do $3^{\circ}$ e $7^{\circ}$ dias de pós-operatório, os vazamentos ocorreram com menor pressão de insuflação nos grupos de animais do terceiro dia, tanto do GP como do GC. Os valores médios de resistência à insuflação de ar observados nos dois grupos estão representados na Figura 6. Na análise estatística desses resultados, não se observou diferença estatisticamente significante nas pressões entre os grupos no período de avaliação do $3^{\circ}$ dia $(p=0,074)$ e no $7^{\circ}$ dia $(p=0,852)$.

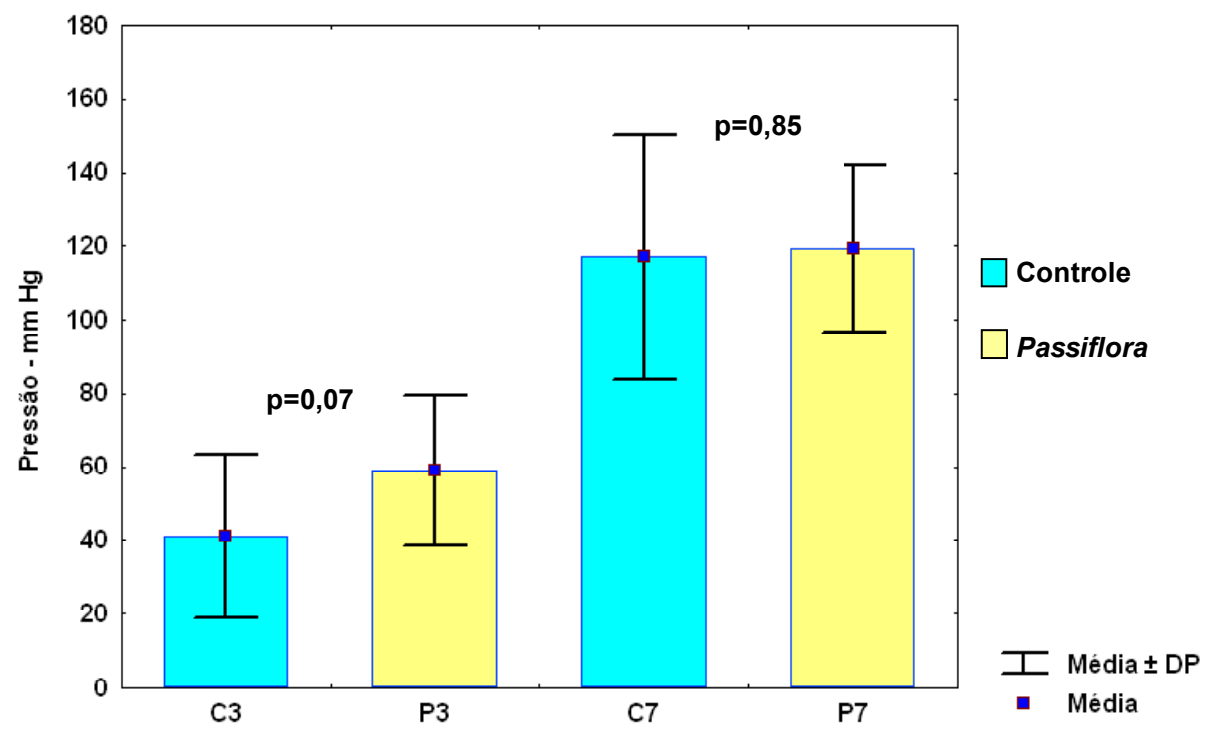

FIGURA 6 - Valores médios da pressão de ruptura dos grupos GC e GP no $3^{\circ}$ e $7^{\circ}$ dia P.O. 


\section{Avaliação microscópica}

A Tabela 2 apresenta a quantificação da intensidade das variáveis histológicas das inflamações aguda e crônica dos grupos GC e GP no $3^{\circ}$ e $7^{\circ}$ dias de P.O.

TABELA 2 - Intensidade da reação inflamatória aguda e crônica das gastrorrafias do GC e GP no $3^{\circ} \mathrm{e}$ $7^{\circ}$ dias de P.O.

\begin{tabular}{lcccccc}
\hline \multirow{2}{*}{$\begin{array}{l}\text { Variáveis } \\
\text { Histológicas }\end{array}$} & $\begin{array}{c}\text { Controle } \\
\text { Dias }\end{array}$ & $\begin{array}{c}\mathbf{7} \\
\text { Dias }\end{array}$ & $\begin{array}{c}\mathbf{3} \\
\text { Dias }\end{array}$ & $\begin{array}{c}\mathbf{7} \\
\text { Dias }\end{array}$ & GC3×GP3 & GC7×GP7 \\
\hline Reação Inflamatória & & & & & 0,733 & 0,111 \\
$\quad$ Aguda & 0 & 2 & 0 & 3 & & \\
$\quad$ Ausente & 0 & 0 & 0 & 4 & & \\
$\quad$ Moderada & 8 & 8 & 7 & 3 & & \\
$\quad$ Intensa & 2 & 0 & 3 & 0 & & \\
Reação Inflamatória & & & & & 0,705 \\
Crônica & & & & & & \\
$\quad \begin{array}{l}\text { Ausente } \\
\quad \text { Dicreta }\end{array}$ & 8 & 0 & 6 & 0 & & \\
$\quad$ Moderada & 2 & 8 & 4 & 9 & & \\
$\quad$ Intensa & 0 & 2 & 0 & 1 & & \\
Teste de Mann-Whitney & 0 & 0 & 0 & 0 & & \\
\hline
\end{tabular}

p - Nível de significância estatística

Como pode ser observado na Tabela 3, a qual apresenta a quantificação da intensidade das variáveis histológicas necrose isquêmica e reação gigantocelular dos grupos GC e GP no $3^{\circ}$ e $7^{\circ}$ dias de P.O., não foram observadas diferenças significativas entre os grupos.

TABELA 3 - Intensidade de necrose isquêmica e reação gigantocelular dos grupos $\mathrm{GC}$ e $\mathrm{GP}$ no $3^{\circ} \mathrm{e}$ $7^{\circ}$ dias de P.O.

\begin{tabular}{|c|c|c|c|c|c|c|}
\hline \multirow{2}{*}{$\begin{array}{l}\text { Variáveis } \\
\text { Histológicas }\end{array}$} & \multicolumn{2}{|c|}{ Controle } & \multicolumn{2}{|c|}{ Passiflora } & \multicolumn{2}{|c|}{ P (inter-grupos) } \\
\hline & $\begin{array}{c}3 \\
\text { Dias }\end{array}$ & $\begin{array}{c}7 \\
\text { Dias }\end{array}$ & $\begin{array}{c}3 \\
\text { Dias }\end{array}$ & $\begin{array}{c}7 \\
\text { Dias }\end{array}$ & GC3 $\times$ GP3 & GC7 $\times$ GP7 \\
\hline Necrose Isquêmica & & & & & 1,000 & 0,571 \\
\hline Aguda & 0 & 1 & 1 & 1 & & \\
\hline Ausente & 9 & 5 & 8 & 6 & & \\
\hline Moderada & 1 & 2 & 1 & 3 & & \\
\hline Intensa & 0 & 2 & 0 & 0 & & \\
\hline $\begin{array}{l}\text { Reação } \\
\text { Gigantocelular }\end{array}$ & & & & & 1,000 & 0,545 \\
\hline Ausente & 10 & 0 & 10 & 0 & & \\
\hline Dicreta & 0 & 4 & 0 & 6 & & \\
\hline Moderada & 0 & 4 & 0 & 2 & & \\
\hline Intensa & 0 & 2 & 0 & 2 & & \\
\hline
\end{tabular}

$\mathrm{p}$ - Nível de significância estatística

\section{Proliferação fibroblástica}

No $3^{\circ}$ dia de pós - operatório, a proliferação fibroblástica no GC foi classificada como ausente nos 10 ratos. No GP foi classificada também como ausente em todos os 10 animais. Não houve diferença estatisticamente significante entre os dois grupos $(\mathrm{p}=1.000)$.

No $7^{\circ}$ dia de pós-operatório, a proliferação fibroblástica no GC foi classificada como discreta em sete ratos; moderada em três (Figura 7). No GP foi classificada como moderada em seis ratos; intensa em quatro (Figura 8). Houve diferença significativa entre os dois grupos $(\mathrm{p}=0,002)$.

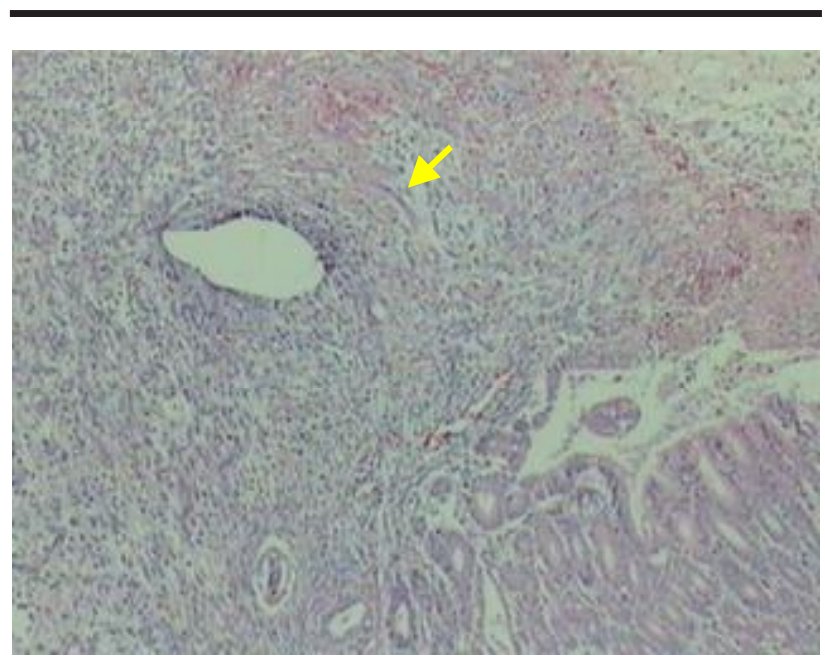

FIGURA 7 - Fotomicrografia do rato do grupo GC7. Observa-se proliferação fibroblástica discreta (M.O., coloração H.E. aumento de 100X). Nota: Fibroblasto (seta)

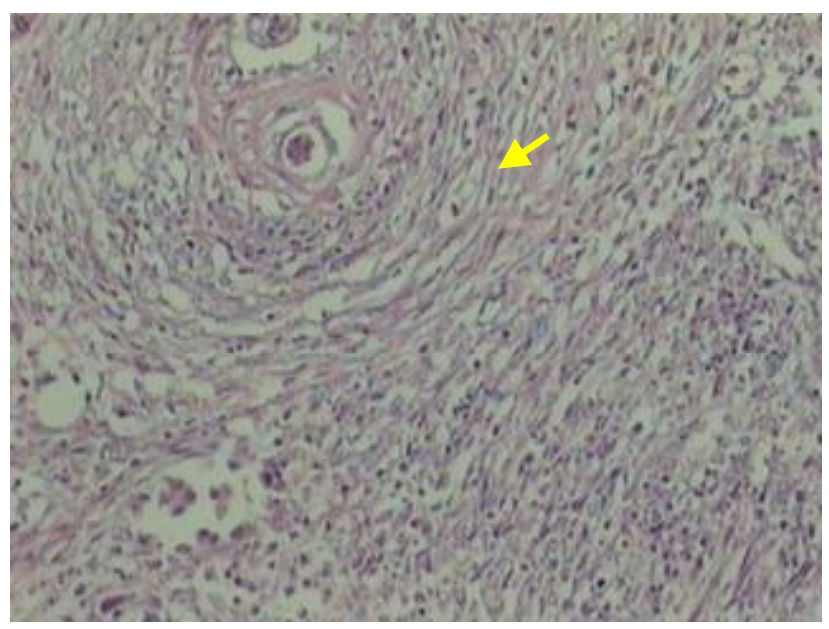

FIGURA 8 - Fotomicrografia do rato do grupo GP7. Observa-se proliferação fibroblástica intensa (M. O. coloração H.E., aumento de 100X). Nota: Fibroblasto (seta)

A Tabela 4 apresenta a quantificação da intensidade das variáveis histológicas proliferação fibroblástica e fibrose (colagenização) dos grupos GC e GP no $3^{\circ}$ e $7^{\circ}$ dias de P.O. Não houve diferença entre os grupos.

A Tabela 5 apresenta a quantificação da intensidade das variáveis histológicas reepitalização e coaptação das bordas dos grupos GC e GP no $3^{\circ}$ e $7^{\circ}$ dias de P.O. Também não foram observadas diferenças significativas entre os 
TABELA 4 - Intensidade de proliferação fibroblástica e fibrose (colagenização) dos grupos GC e GP no $3^{\circ}$ e $7^{\circ}$ dias P.O.

\begin{tabular}{|c|c|c|c|c|c|c|}
\hline \multirow{2}{*}{$\begin{array}{l}\text { Variáveis } \\
\text { Histológicas }\end{array}$} & \multicolumn{2}{|c|}{ Controle } & \multicolumn{2}{|c|}{ Passiflora } & \multicolumn{2}{|c|}{ P (inter-grupos) } \\
\hline & $\begin{array}{c}3 \\
\text { Dias }\end{array}$ & $\begin{array}{c}7 \\
\text { Dias }\end{array}$ & $\begin{array}{c}3 \\
\text { Dias }\end{array}$ & $\begin{array}{c}7 \\
\text { Dias }\end{array}$ & GC3 $\times$ GP3 & GC7 $\times$ GP7 \\
\hline $\begin{array}{l}\text { Proliferação } \\
\text { Fibroblástica }\end{array}$ & & & & & 1,000 & $0,002^{*}$ \\
\hline Aguda & 10 & 0 & 10 & 0 & & \\
\hline Ausente & 0 & 7 & 0 & 0 & & \\
\hline Moderada & 0 & 3 & 0 & 6 & & \\
\hline Intensa & 0 & 0 & 0 & 4 & & \\
\hline $\begin{array}{l}\text { Fibrose } \\
\text { (Colagenização) }\end{array}$ & & & & & 1,000 & 0,450 \\
\hline Ausente & 10 & 6 & 10 & 2 & & \\
\hline Dicreta & 0 & 4 & 0 & 8 & & \\
\hline Moderada & 0 & 0 & 0 & 0 & & \\
\hline Intensa & 0 & 0 & 0 & 0 & & \\
\hline
\end{tabular}

TABELA 5 - Intensidade de reepitelização e coaptação da mucosa nas gastrorrafias dos grupos GC e GP no $3^{\circ}$ e $7^{\circ}$ dias de P.O.

\begin{tabular}{|c|c|c|c|c|c|c|}
\hline \multirow{2}{*}{$\begin{array}{l}\text { Variáveis } \\
\text { Histológicas }\end{array}$} & \multicolumn{2}{|c|}{ Controle } & \multicolumn{2}{|c|}{ Passiflora } & \multicolumn{2}{|c|}{ P (inter-grupos) } \\
\hline & $\begin{array}{c}3 \\
\text { Dias }\end{array}$ & $\begin{array}{c}7 \\
\text { Dias }\end{array}$ & $\begin{array}{c}3 \\
\text { Dias }\end{array}$ & $\begin{array}{c}7 \\
\text { Dias }\end{array}$ & GC3 $\times$ GP3 & GC7 $\times$ GP7 \\
\hline Reepitalização & & & & & 1,000 & 0,677 \\
\hline Ausente & 10 & 3 & 10 & 1 & & \\
\hline Parcial & 0 & 6 & 0 & 9 & & \\
\hline Completa & 0 & 1 & 0 & 0 & & \\
\hline Coaptação das bordas & & & & & 1,000 & 0,705 \\
\hline Ausente & 10 & 0 & 10 & 0 & & \\
\hline Parcial & 0 & 9 & 0 & 10 & & \\
\hline Completa & 0 & 1 & 0 & 0 & & \\
\hline
\end{tabular}

$\mathrm{p}$ - Nível de significância estatística

grupos.

A Tabela 6 apresenta a quantificação da intensidade das variáveis histológicas neoformação capilar e extensão da inflamação dos grupos GC e GP no $3^{\circ}$ e $7^{\circ}$ dias de P.O. Também não foram observadas diferenças significativas entre os grupos.

\section{Discussão}

Hoehne $^{8}$, Corrêa ${ }^{9}$, Balbachas ${ }^{10}$, descrevem a Passiflora edulis como sendo usada pela população com finalidade terapêutica. A utilização das suas folhas para a obtenção do extrato bruto é um processo já bem estabelecido e difundido. Admite-se que o extrato obtido das folhas seja mais eficaz que o produzido de flores, frutos, sementes e raízes.

O extrato é utilizado na sua forma alcoólica de modo intraperitoneal, tópico sobre áreas de processos inflamatórios (traumáticos ou infecciosos) ou sobre feridas cutâneas ou mucosas. É também ingerido na forma de chá ou gotas (solução de concentrações variadas preparadas por manipulação a partir do extrato bruto da planta).

Hoehne $^{8}$ demonstraram que o extrato etanólico (EE)
TABELA 6 -Intensidade de neoformação capilar e extensão da inflamação das gastrorrafias dos grupos GC e GP analisadas no $3^{\circ}$ e $7^{\circ}$ dias de P.O.

\begin{tabular}{|c|c|c|c|c|c|c|}
\hline \multirow{2}{*}{$\begin{array}{l}\text { Variáveis } \\
\text { Histológicas }\end{array}$} & \multicolumn{2}{|c|}{ Controle } & \multicolumn{2}{|c|}{ Passiflora } & \multicolumn{2}{|c|}{ P (inter-grupos) } \\
\hline & $\begin{array}{c}3 \\
\text { Dias }\end{array}$ & $\begin{array}{c}7 \\
\text { Dias }\end{array}$ & $\begin{array}{c}3 \\
\text { Dias }\end{array}$ & $\begin{array}{c}7 \\
\text { Dias }\end{array}$ & GC3 $\times$ GP3 & GC7 $\times$ GP7 \\
\hline Neoformação Capilar & & & & & 0,705 & 0,290 \\
\hline Aguda & 8 & 0 & 7 & 0 & & \\
\hline Ausente & 2 & 5 & 3 & 3 & & \\
\hline Moderada & 0 & 4 & 0 & 4 & & \\
\hline Intensa & 0 & 1 & 0 & 3 & & \\
\hline Extensão da Inflamação & & & & & 1,000 & 1,000 \\
\hline Mucosa & 0 & 0 & 0 & 0 & & \\
\hline Submucosa & 0 & 0 & 0 & 0 & & \\
\hline Muscular & 0 & 0 & 1 & 0 & & \\
\hline Serosa & 10 & 10 & 9 & 10 & & \\
\hline
\end{tabular}

p - Nível de significância estatística

das folhas de Passiflora edulis apresentam atividades analgésica pela redução do número de contorções abdominais em camundongos. Também demonstraram diminuição do edema de orelha em camundongos e a redução do edema de pata em ratos, sugerindo a presença de compostos químicos relacionados com a atividade analgésica e antiinflamatória, característica de antiinflamatórios não esteróidais (AINES). Os AINES diminuem a dor por inibição da biossíntese de eicosanóides, mediadores de processos inflamatórios ${ }^{11}$.

Escolheram-se os períodos de morte dos ratos no $3^{\circ}$ e $7^{\circ}$ dias de pós-operatório para que se pudesse estudar a cicatrização gástrica em suas fases críticas. Nessas fases o processo inflamatório é principalmente agudo e a taxa global de degradação de colágeno é maior do que a taxa de síntese, principalmente até o $5^{\circ}$ dia de pós-operatório, quando a neovascularização atinge um pico. As fibrilas do colágeno tornam-se mais abundantes e começam a constituir pontes sobre a incisão.

O $7^{\circ}$ dia de pós-operatório foi escolhido porque é em torno desta fase que ocorre a maioria das principais complicações das anastomoses gástricas, como o extravasamento anastomótico e a ruptura da parede gástrica, que se associam a morbidade e mortalidade significativas ${ }^{11}$.

A administração intraperitoneal do extrato etanólico em ratos foi bem tolerada e produziu efeitos expressivos na sutura gástrica. A via peritoneal foi preferida à via oral por diversos motivos. Segundo Benet et al. ${ }^{12}$ esta via tem as seguintes vantagens: a) maior e mais rápida absorção de substâncias ativas extraídas da planta pela via intraperitoneal; b) ao metabolismo hepático (conseqüência da primeira passagem); e c) evita a inativação dos princípios ativos pelas secreções da mucosa do sistema digestório. A utilização de dose única de $250 \mathrm{mg} / \mathrm{ml}$, no dia da opperação, nos ratos do GP, foi baseada no estudo do efeito antiinflamatório, analgésico e antipirético do extrato etanólico de folhas de Passiflora edulis realizados por Hoehne ${ }^{8}$, cujos resultados sugerem a presença de compostos com atividades analgésica e antiinflamatória semelhantes às encontradas nos antiinflamatórios não esteróides (AINES), como a indometacina, considerado como o mais importante inibidor da biossíntese dos eicosonóides, Brennan et al. ${ }^{3}$, observaram efeito 
favorável dos AINES sobre a cicatrização anastomótica por aumentarem a produção de colágeno. O efeito antiprostaglandina, principalmente de natureza seletiva antiprostasglandina E2, favorece a cicatrização anastomótica ${ }^{14}$. Foi realizada dose única e não diária devido a dificuldade no manejo dos animais e no sentido de poupar-lhes de agressões de ordem física, psíquica e infecciosa, e outras, capazes de perturbar-lhes a homeostasia e com isso prejudicar os objetivos do trabalho.

\section{Avaliação macroscópica}

Não se observaram sinais de peritonite provocada por fístulas ou abscessos intrabdominais em ambos os grupos. Segundo Kuzu, Koskoy, Kale et al. ${ }^{14}$, a intensidade das aderências é medida indireta da presença de complicações anastomóticas e, por conseqüência, também medida indireta da cicatrização das anastomoses. Quando ocorre deiscência há vazamento de conteúdo intestinal dentro da cavidade peritoneal. Para a contenção de tal vazamento o organismo responde com bloqueio do local mediante a migração de estruturas intra-abdominais e formação de aderências. Neste estudo, o extrato de Passiflora edulis foi bem tolerado na cavidade peritoneal, pois não houve aumento de aderências em ambos os grupos. Em sua maioria, as aderências foram frouxas ou com bridas entre as estruturas vizinhas e a linha de sutura gástrica ou com a parede abdominal, não ultrapassando o grau 2 do índice de Knightly ${ }^{4}$.

\section{Avaliação tensiométrica}

Os parâmetros mecânicos são utilizados freqüentemente em estudos de cicatrização de suturas intestinais devido à sua confiabilidade. Os autores têm opiniões diferentes quanto ao melhor método para avaliar a resistência da anastomose. A força de explosão representa a força exercida pela pressão intra-luminal, que, segundo Koruda e Rolandelli ${ }^{15}$, seria a força que naturalmente atua sobre as suturas intestinais. A força de ruptura representa a força aplicada perpendicularmente à linha de sutura, até sua ruptura. É um método mecânico de avaliação de cicatrização de anastomose no período tardio, após o $14^{\circ}$ dia de pós-operatório, quando se pode utilizar a determinação da resistência à tração linear de uma tira de tecido isolado.

Neste estudo optou-se pela utilização da medida da pressão de ruptura, mesmo sabendo-se, segundo Ikeuchi et al. ${ }^{16}$, poder ser influenciada por erros técnicos do cirurgião. Porém, baseado em trabalhos de Gottrup ${ }^{17}$, Zederfeldt ${ }^{18}$, Czeczko $^{6}$, Wendler ${ }^{19}$ e Porcides ${ }^{20}$, a pressão de ruptura é parâmetro fisiológico importante na avaliação de uma anastomose, principalmente nos primeiros sete dias de pós-operatório, porque dá idéia da vedação da anastomose em sua totalidade.

No presente trabalho, observou-se resistência à insuflação de ar atmosférico baixa no $3^{\circ}$ dia de pós-operatório tanto para o GP quanto para o GC, a qual elevou-se no $7^{\circ}$ dia, porém com níveis semelhantes nos dois grupos. De acordo com Herrmann et al. ${ }^{21}$ e Jiborn et al. ${ }^{22}$ esses resultados confirmam o padrão conhecido de baixa resistência à insuflação de ar na fase de maior colagenólise, isto é, $3^{\circ}$ dia de pós-operatório. Nos dois grupos estudados, a análise estatística demonstrou que a resistência à insuflação de ar foi significativamente menor no $3^{\circ}$ dia de pós-operatório em comparação ao $7^{\circ}$. No $3^{\circ}$ dia P.O observou-se que a pressão média de resistência à insuflação de ar no GP foi superior ao GC, porém esta diferença não atingiu nível de significância estatistística $(p=0,074)$. No $7^{\circ}$ dia P.O não foi observada diferença significativa entre os grupos GC e GP quanto à pressão média no teste de insuflação de $\operatorname{ar}(\mathrm{p}=0,852)$. Esses resultados sugerem que o extrato etanólico de Passiflora edulis não altera a resistência das gastrorrafias em ratos, apesar de observar-se certa tendência de aumento da resistência das gastrorrafias, principalmente na fase precoce da cicatrização.

\section{Avaliação microscópica}

$\mathrm{Na}$ análise da variável reação inflamatória aguda o parâmetro classificatório moderado foi dominante nos dois grupos, o qual, segundo Nomura ${ }^{23}$, favorece o processo de cicatrização pelo desenvolvimento do tecido de granulação, ocorrendo o contrário se o processo inflamatório for ausente ou excessivo, pois pode atrapalhar o adequado suprimento sangüíneo pelo comprometimento da microcirculação e ainda irá inibir a proliferação de fibroblastos. A intensidade da reação inflamatória crônica, entre os dois grupos estudados, foram predominantemente classificadas de ausentes a discretas. Na necrose isquêmica foi discreta e ausentes na neoformação capilar, reação gigantocelular, proliferação fibroblástica, fibrose (colagenização), reepitelização, coaptação das bordas. Observou-se que não houve diferença estatística na análise dos parâmetros das variáveis histológicos nos subgrupos estudados na fase inicial. Estas alterações são condizentes com esta fase da cicatrização, tendo sido relatadas em trabalhos que fizeram comparação da cicatrização com o uso de materiais de sutura como nos estudos de Tabushi ${ }^{24}$, Nomura ${ }^{23}$ e Porcides ${ }^{20}$.

No $7^{\circ}$ dia de pós-operatório, os cortes histológicos analisados apresentaram regeneração da mucosa totalmente completada, com tendência para redução da reação inflamatória aguda nos dois grupos de moderada a discreta, principalmente no subgrupo Passiflora edulis, porém não houve diferença estatisticamente significante $(\mathrm{p}=0,112)$. O reparo fibroso cicatricial mostrou, nos dois grupos, neoformação capilar moderada, principalmente no GP, porém não apresentaram diferença estatisticamente significante ( $p=0,290)$; reação inflamatória crônica e necrose isquêmica apresentaram-se discretas. A proliferação fibroblástica e a colagenização tiveram aumento, sendo a primeira significativamente maior no $\mathrm{GP}(\mathrm{p}=0,002)$, enquanto a colagenização teve aumento discreto nos dois grupos.

\section{Conclusão}

O uso intraperitoneal do extrato de Passiflora edulis influencia favoravelmente a cicatrização das gastrorrafias em ratos pelo aumento da proliferação fibroblástica no $7^{\circ}$ dia de pós-operatório. 


\section{Referências}

1. Madden JW. Cicatrização das Feridas: Aspectos Clínicos e Biológicos. In: Sabiston Jr DC. Tratado de Cirurgia. Rio de Janeiro: Interamericana; 1964: 254- 73.

2. Robbins SL, Kumar V. Inflammation and Repair. In: Robbins SL, Cotran RS, Kumar V, Schoen FJ. Robbins Pathologic Basis of Disease. $5^{\text {nd }}$. Philadelphia: WB Saunders; 1994.

3. Brennan SS, Foster ME, Morgan A, Leaper DJ Prostaglandins in colonic anastomotic healing. Dis. Colon Rectum. 1984; 27: 723-5.

4. Knigthly JJ, Agostino D, Cliffton EE. The effect of fibrinolysin and heparin on the formation of peritoneal adhesions. Surgery. 1962;52:250-8.

5. Warde PJ. Anastomose colorretal experimental em um e em dois planos de sutura: estudo comparativo [Tese - Doutorado] São Paulo: Universidade de São Paulo - Faculdade de Medicina; 1972.

6. Czeczko NG. Estudo comparativo entre as anastomoses colorretais mecânicas com grampeador e com anel biodegradável no reto extraperitoneal de cães. [Tese - Doutorado] Curitiba:. Universidade Federal do Paraná - Setor de Ciências da Saúde; 1993.

7. Cotran RS. Robbins basis of disease. Philadelphia: WB. Saunders; 1999.

8. Hoehne FC. Plantas e substâncias vegetais tóxicas e medicinais. São Paulo: Instituto de Botânica; 1978.

9. Correa MP. Dicionário das plantas úteis do Brasil e das exóticas cultivadas. Rio de Janeiro, R. J: Ministério da Agricultura e IDDF (editors.); 1984; 6.

10. Balbachas A. A flora nacional na medicina doméstica. São Paulo: A edificação do lar (editor); 1991.

11. Cohen IK, Diegelmann RF, Crossland MC. Os cuidados com a ferida. In: Sabiston Jr., D. C. Tratado de Cirurgia. Rio de Janeiro: Interamericana; 1986.

12. Benet LZ, Kroetz DL, Sheiner LB. "Pharmacokinetics: The dynamics of drug absorption, distribution, and elimination". In: Molinoff PB, Ruddon RW editors. The pharmacological basis of therapeutics. McGraw-Hill; 1996.

13. Thornton FJ, Barbul A. Healing in the gastrointestinal tract. In: Barbul A. Wound healing. Surg Clin North Am.1997; 77: 547-70.
14. Kuzu MA, Koskoy C, Kale T, Demiprence E, Renda N. Experimental study of the effect of preoperative 5fluorouracil on the integrity of colonic anastomoses. $\mathrm{Br}$ J Surg. 1998;85: 236-9.

15. Koruda MJ, Rolandelli RH. Experimental studies on the healing of colonic anastomoses. J Surg Res .1990;48: 504-15.

16. Ikeuchi D, Onodera H, Aung T, Kan S, Kawamoto H, Iamamura M, Maetani S. Correlation of tensile strength with bursting pressure in the evaluation of intestinal anastomoses. Dig. Surg. 1999; 16: 478-85.

17. Gottrup F. Healing of incisional wounds in stomach and duodenum. A biomechanical study. Am JSurg. 1980;140: 296-301.

18. Zederfeldt B. Anastomotic healing. In: Eigler, FW, Gross W, Vogt E. Die anastomose am gastrointestinaltrakt. TM - Verlag Hameln. 1990.

19. Wendler LE. Estudo experimental comparativo da cicatrização de suturas jejunoileais em cães, utilizando o fio de poliglecaprone 25 [Tese - Doutorado]. Curitiba: Faculdade Evangélica de Medicina do Paraná; 1995.

20. Porcides RD. Estudo comparativo entre os fios de polipropileno e glicomer 60 na gastrorrafia em cães [Dissertação - Mestrado]. Curitiba: Faculdade Evangélica do Paraná; 2002.

21. Hermann JB; Woodward SC, Pulaski EJ. Healing of colonic anastomoses in the rat. Surg Gynecol Obst 1964;119: 269-75.

22. Jiborn H, Ahonen J, Zederfeldt B. Healing of experimental colonic anastomoses: The effect of suture technic on collagen concentratrations in the colonic wall. Am J Surg. 1978; 135:333-40.

23. Nomura LM. Estudo experimental comparative da cicatrização de sutura em ceco de ratos, utilizando os fios de polipropileno, poligrecapone 25 e glicomer 60 [Dissertação - Mestrado]. São Paulo: Instituto de Pesquisas Médicas, Faculdade Evangélica de Medicina; 1999.

24. Tabushi FI. Cecorrafia em plano único com polipropileno e com poliglecaprone 25: estudo comparativo em ratos [Dissertação - Mestrado].Curitiba: Instituto de Pesquisas Médicas, Faculdade Evangélica de Medicina;1998

\section{Correspondência}

José Ribamar Sousa da Silva

Hospital Universitário Presidente Dutra

Rua Barão de Itapary, 227 - Centro - São Luis-MA

CEP: $65020-070$

Tel: (98) 2109-1000
Conflito de interesse: nenhum

Fonte de financiamento: Capes

Recebimento: 19/01/2005

Revisão: 21/05/2005

Aprovação: 27/06/2006

\section{Como citar este artigo}

Silva JRS, Campos ACL, Ferreira LM, Aranha Jr AA, Zago-Filho LA, Bertoli LC, Ferreira M, Trubian PS, Freitas ACT. Avaliação da ação da Aroeira (Schinus Efeito do extrato da Passiflora edulis na cicatrização de gastrorrafias em ratos: estudo morfológico e tensiométrico.Acta Cir Bras. [periódico na Internet] 2006; Suppl 2:52-60. Disponível em URL: http://www.scielo.br/acb 